Intelligent Integrated Management for Telecommunication Networks

Antonio Martín, Carlos León, Félix Biscarri

\title{
Intelligent Integrated Management for Telecommunication Networks
}

\author{
Antonio Martín, Carlos León, Félix Biscarri \\ Electronic Technology Department, Seville University, Seville, 41012, Spain \\ toni@us.es,cleon@us.es \\ doi: 10.4156/ijact.vol2.issue2.15
}

\begin{abstract}
As the size of communication networks keeps on growing, faster connections, cooperating technologies and the divergence of equipment and data communications, the management of the resulting networks gets additional important and time-critical. More advanced tools are needed to support this activity. In this article we describe the design and implementation of a management platform using Artificial Intelligent reasoning technique. For this goal we make use of an expert system. This study focuses on an intelligent framework and a language for formalizing knowledge management descriptions and combining them with existing OSI management model. We propose a new paradigm where the intelligent network management is integrated into the conceptual repository of management information called Managed Information Base (MIB). This paper outlines the development of an expert system prototype based in our propose GDMO+ standard and describes the most important facets, advantages and drawbacks that were found after prototyping our proposal.
\end{abstract}

Keywords: Expert System, GDMO, MIB, OSI, TMN, Artificial Intelligent, Network Management

\section{Introduction}

As communication networks and distributed processing systems are gaining importance their reliable operation is getting vital to businesses. Networks are getting bigger and more complex. These have a problem as they have been built over a long time and consist of very heterogeneous equipment which has to work together. Traditional network management models are inadequate to support this large demand of service. In the traditional manager-agent network management architecture, the agent is kept as simple as possible, only tasked with device status report and update, while the burden of management and data processing resides with the manager.

It is necessary to develop new models, which offer more possibilities. We believe that distributing intelligence to management objects is an inevitable trend in network management and one that is critical to the success of future network management designs. The objective of this work is to improve insight and understanding of network management, and present an alternative network management model. We propose a new evolution called Integrated Management Expert Systems. For this aim, an extension of OSI management framework specifications language has been added and investigated in this study.

In modern network elements management information is increasingly stored in a distributed manner locally with the network elements into Management Information Base (MIB) databases. These databases contain all relevant configuration data and the dynamic state data (measurements and alarms) in a standardized format. We study a technique which integrates the knowledge base of expert system within the MIB used to manage a network. A new property named RULE has been added in the MIB, which gathers important aspects of the facts and the knowledge base of the embedded expert system. By integrating the knowledge base in resources specifications, expert system has the power to provide diagnosis of fault network, which can assist engineering trainees, inspectorate staff and professional.

In this study we will examine the management network, including the concepts, major approaches, and management models. We propose a new management model Integrated Management Expert System and an extension of standard called Extended GDMO or simply GDMO+, for the incorporation of the management expert rules. Next will be examined the design and development of the expert system named NOMOS+. It starts with specific applications and work on expert systems in similar fields. From there, we present the concept the formulation of the NOMOS+ system design proposal and also an outline of the various stages in the system development cycle. A detailed description of the 
various modules of NOMOS+ is also given. Next section summarizes the performance of NOMOS+ and the results of the research. Finally we outline the conclusion and future works.

\section{Networks Management and Expert Systems}

The basic network management model is a starting point for understanding network management. There are two main standards for controlling telecommunications equipment: TMN and SNMP. Both network management systems operate using client/server architecture. SNMP standards are defined in a series of documents, called request for comments or RFCs proposed by the Internet Engineering Task Force (IETF) [1] and Telecommunications Management Network TMN is introduced by the ITU-T (the former CCITT) [2][3]. Of these two TMN is gaining popularity for large complex networks.

In private network environment, SNMP enjoys near-universal support. In the public environment, however, a more heterogeneous mix of de facto telecommunications industry standards has prevailed, with a move toward TMN support. Moreover TMN was the first who started, as part of its Open Systems Interconnection (OSI) program, the development of the architecture for network management. The OSI management environment consists of tools and services need to control and supervise the management networks.

In network management a main concept is the managed object, which is an abstract view of a logical o physical resource to be managed in the network. Managed objects provide the necessary operations for the administration, monitoring and control of the telecommunications network [4]. These operations are realized through the use the Common Management Information Protocol (CMIP) [5]. This is a network management protocol built on the OSI communication model. The related Common Management Information Services (CMIS) defines services for accessing information about network objects or devices, controlling them, and receiving status reports from them. For a specific management system, the management process involved will take on one of two possible roles [6], Figure 1:

- A Manager or Manager Role is an element that provides information to users, issues requests to devices in a network, receives responses to the requests and receives notifications. These notifications are unsolicited information from devices in the network concerning the status of the devices.

- Agent or Agent Role is an unit that is part of a device in the network that monitors and maintains status about that device. It can act and respond to requests from a manager and can provide unsolicited information (or notifications) to a manager.

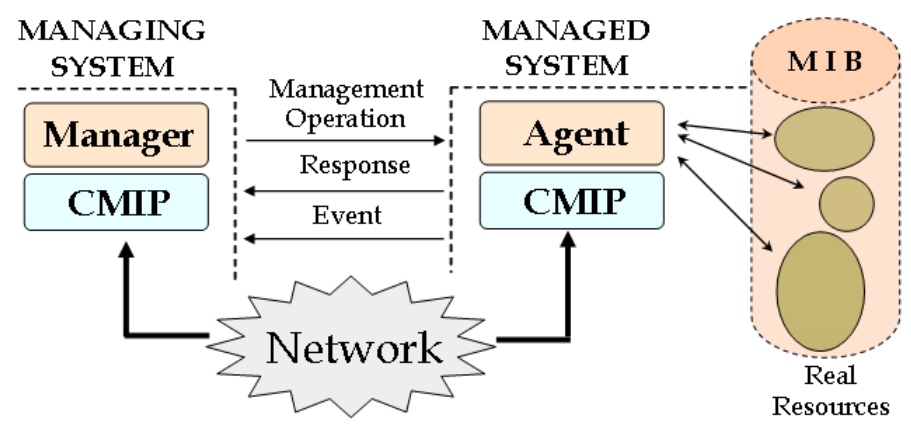

Figure 1. OSI architecture

These managed objects are defined according the ISO Guidelines for Definition of Managed Objects (GDMO) [7]. GDMO language uses the object orient programming and defines how network objects and their behavior are to be specified, including the syntax and semantics. This standard has been standardized by ITU (International Telecommunication Union) in ITU-T X.722 and is now widely used to specify interfaces between different components of the TMN architecture. GDMO properties values types are described using the abstract syntax notation one (ASN.1) [8]. ASN.1 describes an abstract syntax for data types and values.

Nowadays there are different domains of application of the expert systems in topics related with the network management. OSI classifies the systems management activities into five functional areas: fault 
management, accounting management, configuration management, performance management and, security management [9]. We can categorize the expert systems used in network management within these five groups [10]. Some expert system examples are Max \& Opti, ANSWER, Trouble Locator, and CRITTER in fault diagnosis area, ESS-ES, ECXpert, and APRI in accounting management area, ACE, XCON, SMCS, and EXSim in configuration management area, TASA, NETTRA, and Scout in performance management area, NIDES, P-BEST, and NIDX in security management area. In this context the expert system that we have built NOMOS+, would be included in the area of work of the fault management.

After this brief introduction to management elements, we will approach our research in the integration of knowledge management of expert system into MIB in the OSI management model. We are studying the way to integrate the expert knowledge in the management Internet model. Internet management model doesn't use the Object Oriented Programming such as it is used by the OSI model. This is one of the reasons for the Internet model simplicity. The definitions contain objects, specified with ASN.1 macros. In internet model the resources specifications can only be groups of scalar variables and cells tables in spite of not being an Object Oriented Programming model. We can use the tables of the Internet model as classes of the OSI model, where the attributes are the table columns and every file contains an instance of the class. The same as in OSI every object has an OID associated identifier [9].

\section{Including Expert Knowledge in GDMO Description}

Practical experience with GDMO shows that, from an intelligent point of view, the quality of GDMO specifications is not satisfactory. The managed object specifications are incomplete to define the management knowledge of a specific resource. As consequence a new element is necessary. To solve the current problem to undertake an intelligent integrated management we offer an original contribution to include expert rules in the specifications of the network features [11]. To answer these questions, it will be necessary to make changes on the template of the GDMO standard. To formalize the main proposal of the paper, we analyze necessary requirements area to undertake the related aspects with the knowledge integration in the managed objects. We present an extension of the standard GDMO, to accommodate the intelligent management requirements [12].

We propose to extend the Guidelines for Definition of Managed Objects with the following goals: facilitate the normalization and integration of the knowledge base of expert system into resources specifications. These goals will allow to developers specify the storage location and the update method of intelligent managed and provide a way to specify complex managed.

GDMO is organized into templates, which are standard formats used in the definition of a particular aspect of the object. A complete object definition is a combination of interrelated templates. There are nine of these templates: class of managed objects, package, attribute, group of attributes, action, notification, parameter, connection of name and behavior [13]. The set of managed object classes and instances under the control of an agent is know as it's a MIB [14], an abstraction of network resources properties and states for the purpose or management. The MIB, which is specified using the Structure Management Information (SMI) defines the actual objects to be managed [15].

The elements that at the moment form the GDMO standard do not make a reference to the knowledge base of an expert system. Until now the managed objects are not able to use the knowledge that the base of knowledge provides which collects the management operations and control of a management domain. We observe the need to define new structures for those cases in which it is necessary to express the knowledge. To solve this problem we suggest a refinement of the package template. Thus the description of certain aspects of managed object knowledge, e.g. the definition of expert rules, can be supported. We proposed adding a new property in GDMO standard named "rule". This attribute will define all the aspect about the management knowledge in a specific managed object class, Figure 2. 


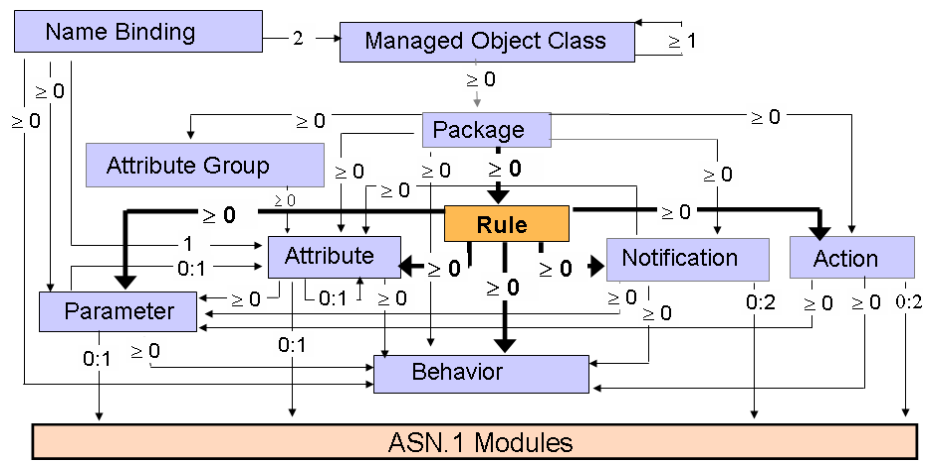

Figure 2. Template relations in GDMO+

Two relationships are essential for the inclusion of knowledge in the component definition of the network: Managed Object Class and Package. GDMO includes the basic template MANAGED OBJECT CLASS, which is always implemented and GDMO also defines an optional template named PACKAGE, which defines a combination of properties for later inclusion in a managed object class template [16].

\subsection{Management Object Class Template}

Classes describe what information and services they provide each manage object and GDMO defines format for this information. This template is used to define the different kinds of objects that exist in the system. The definition of a managed object class is made uniformly in the standard template, eliminating the confusion that may result when different persons define objects of different forms. This way we ensure that the classes and the management expert rules defined in system A can be easily interpreted in system B. Managed object class structure is show here:

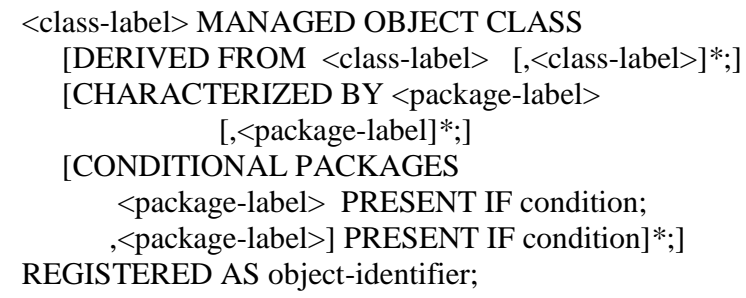

DERIVED FROM clause specifies the superclass or superclasses from which this managed object is derived (inherited). This plays a very important role, when determining the relations of inheritance which makes it possible to reutilize specific characteristics in other classes of managed objects. In addition, a great advantage is the reusability of the object classes and therefore of the expert rules which are defined.

Packages included in the object class definition are identified by the CHARACTERIZED BY and CONDITIONAL PACKAGES clauses. The CHARACTERIZED BY clause identifies the package or packages that are always present when the managed object is included in the system. The CONDITIONAL PACKAGES clause is used to identify those packages that may or may not be included each time the managed object of this class is instantiated. Finally, the REGISTERED AS clause identifies the location of the managed object class on the OSI registration tree.

\subsection{Package Template}

The PACKAGE template is used to specify the characteristics that represent a consistent set of specifications about a network resource. One purpose of the package is to provide a set of re-useable definitions that can be used in several managed object class specifications. All the properties that we 


\section{Intelligent Integrated Management for Telecommunication Networks} Antonio Martín, Carlos León, Félix Biscarri

define in the package will be included later in the Managed Object Class Template, where the package is incorporated. A same package can be referenced by more than one class of managed objects. For each managed objects class, the following information is defined:

1) Attributes: are the types of data supported by the class (managed object).

2) Operations: are the actions supported by the class.

3) The behavior of the managed object.

4) Notifications: are the types of unsolicited information a managed object can send to a manager.

The current template package in GDMO standard is adapted and we add a new feature. In addition to the properties indicated above, we suggest the incorporation of a new property called RULES and its associated template called "RULE", which contains all the specifications of the knowledge base for the expert system. Next definition shows the elements of a package template, in which it is possible to observe the new property RULES.

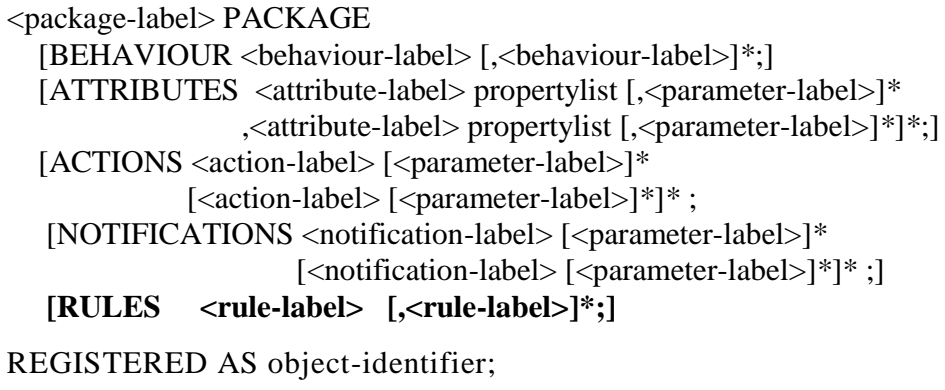

The property RULES allows a treatment similar to the other properties, including the possibility of inheritance of rules between classes. Like the rest of the other properties defined in a package, the property RULE needs a corresponding associated template.

\subsection{Expert Rule Template}

There are a number of different knowledge representation techniques for structuring knowledge in an expert system. The three most widely used techniques are expert rules, semantic nets and frames [17]. For this study we use expert rules. We represented the knowledge in production rules or simply rules. Rules are expressed as IF-THEN statements which are relatively simple, very powerful as well as very natural to represent expert knowledge. A major feature of a rule-based system is its modularity and modifiability which allow for incremental improvement and fine tuning of the system with virtually no degradation of performance.

In our study case the template RULE permits the normalized definition of the specifications of the expert rule to which it is related. This template allows a particular managed object class to have properties that provide a normalized knowledge of a management dominion [18]. The structure of the RULE template is shown here:

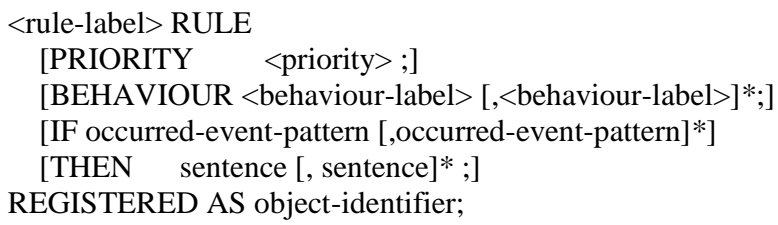

The first element in a template definition is headed. It consists of two sections:

- <rule-label>: This is the name of the management expert rule. Rule definitions must have a unique characterizing name.

- RULE: A key word indicates the type of template, in our case a definition template and the specifications for the management expert rule.

After the head, the following elements compose a normalized definition of an expert rule. 
- BEHAVIOUR: This construct is used to extend the semantics of previously defined templates. It describes the behavior of the rule. This element is common to the others templates of the GDMO standard.

- PRIORITY: This represents the priority of the rule, that is, the order in which competing rules will be executed.

- IF: It contains all the events that must be true to activate a rule. Those events must be defined in the Notification template. The occurrence of these events is necessary for the activation of the rule and the execution of their associated actions. We can add a logical condition that will be applied on the events occurred or their parameters.

- THEN: This gives details of the operations performed when the rule is executed. Those operations must be previously defined in the Action template. These are actions and diagnoses that the management platform makes as an answer to network events occurred.

- REGISTERED AS is an object-identifier: A clause identifies the location of the expert rule on the OSI registration tree. The identifier is compulsory.

\section{Development Work}

In order to determine the suitability of this approach in large network management problems the method should be evaluated with a test case. For this purpose we have developed a prototype for the specifications performed with GDMO+. We used a telecommunications network that belongs to a company the electrical sector (SEVILLANA-ENDESA's a major Spanish power utility) [19]. Part of long-distance traffic in this net is controlled by a wireless intelligent system distributed through-out of this private network, Figure 3.

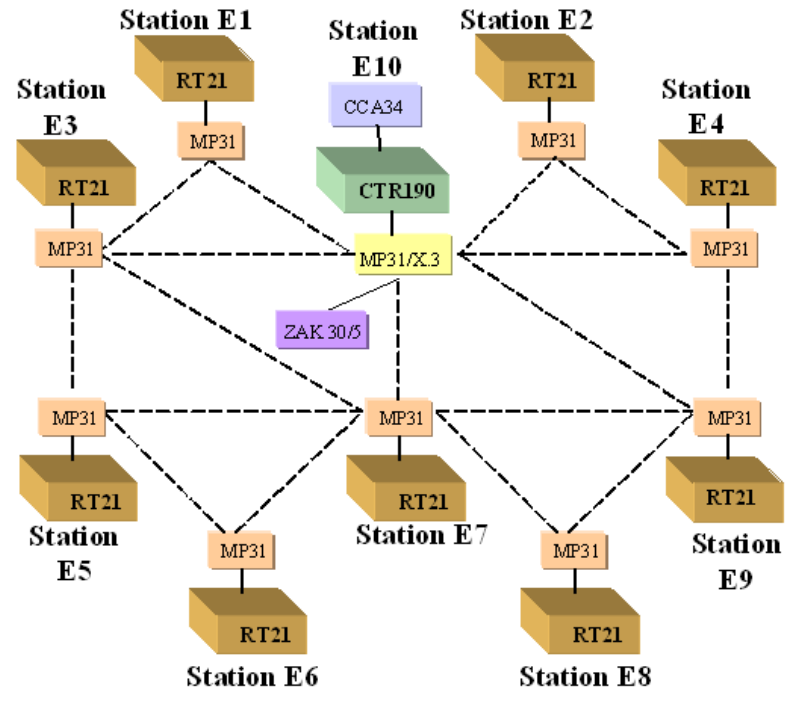

Figure 3. Power Company Network

Expert system is part of the system dedicated to the management this power utility's communications system, which we call NOMOS+ [20]. The intelligent system should meet the following requirements:

1) It should perform non-intrusively. The management activity should not interfere with normal operations of the network. It must only intervene when necessary. Excessive polling wastes bandwidth that could be used for other important services.

2) It should be robust. Management applications should be able to perform even when the network is not fully operational as management is mostly needed in abnormal situations, e.g. when connections are broken.

3) It should scale up well to growing networks. 
Intelligent Integrated Management for Telecommunication Networks Antonio Martín, Carlos León, Félix Biscarri

Next we will study the main elements of the system.

\subsection{The System Architecture}

Our system has three major components: an inference engine, a knowledge base, and a user interface, Figure 4.

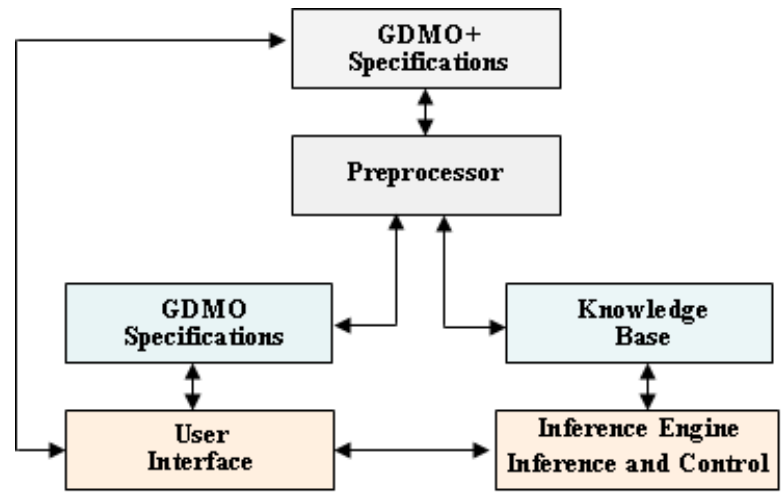

Figure 4. Elements of the prototype NOMOS+

Those elements are briefly discussed in the following [21]:

The inference engine: This is the processing unit that solves any given problems by making logical inferences on the given facts and rules stored in the knowledge base. It defines the managed objects and the expert rules belonging to the Expert System that manages this network. NOMOS+ is implemented in Brightware's ART*Enterprise, an expert system shell. ART*Enterprise is a set of programming paradigms and tools that are focused on the development of efficient, flexible, and commercially deployable knowledge-based systems. Expert system shells simplify developer interactions by eliminating the developer's concern with operating system requirements. Its use can therefore reduce the design and implementation time of a program considerably. By using an existing general purpose tool we were able to build a standard and extensible platform with proven performance and quality. The experience with NOMOS+ is that ART Enterprise is a useful tool for developing expert systems.

The knowledge base: The core of the system, this is a collection of facts and if-then production rules that represent stored knowledge about the problem domain. The knowledge base of our system is a collection of expert rules and facts expressed in the ARTScript programming language ART*Enterprise. The knowledge base contains both static and dynamic information and knowledge about different network re-sources and common failures. The resultant expert system has about 200 rules and it has been employed Workstation to program the expert system. This initial knowledge has been acquired from the experts in the management domain [22]. The knowledge base of our system can be extended by adding new higher level rules and facts.

The user interface controls the inference engine and manages system input and output. It is a set of I/O handling routines for managing the system [23]. This includes tools for browsing the inheritance architecture classes generated by both GDMO and ASN.1 compilers. Moreover our system includes facilities to browse the GDMO classes using a Web browser such as Explorer o Mozilla, Figure 5. 


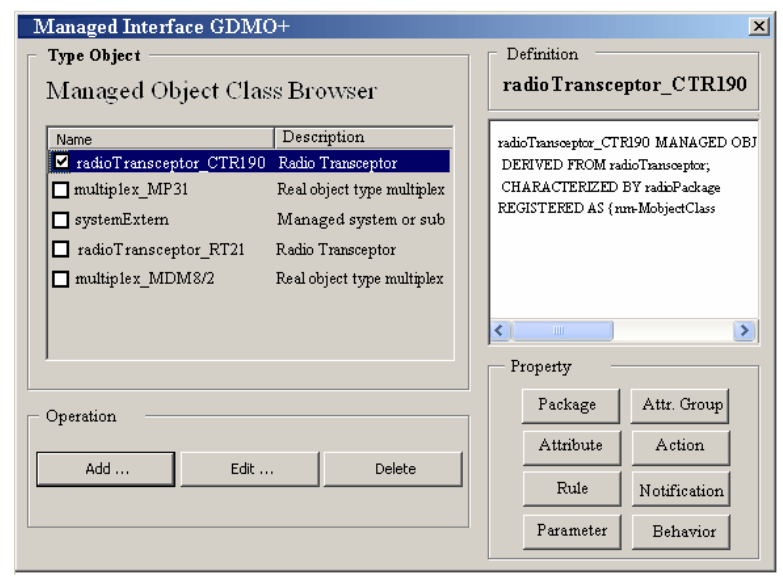

Figure 5. System Object Classes Dialog Box

The user interface is easy to use and enable mobile management in that remote access only requires access to the Internet and a Web browser. Our user interface also contains a preprocessor for parsing GDMO+ specification files. The user interface components named GDMO Managed Object Definition Tool allow administrators to inspect the definitions of management object classes interactively. GDMO Schema Browser simplifies the task of defining MO definitions. When new knowledge is uncovered, it will need to be incorporated into the system to keep it updated. The user interface GDMO Template Editor and Parser allows to modifying or including new expert management rules in the managed objects definition, Figure 6.

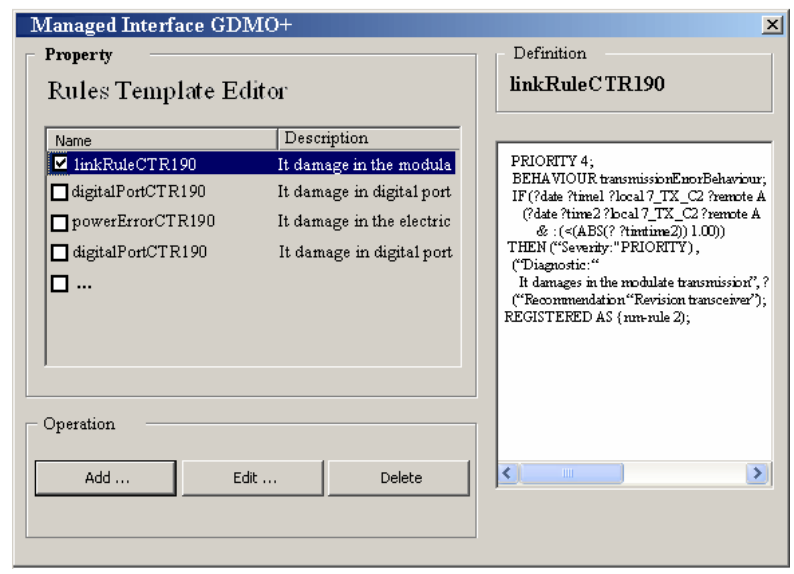

Figure 6. System Object Classes Update

\section{Communication Supervision System}

Detection mechanisms are implemented real-time in our prototype and have been embedded with the network elements, network protocols and devices. NOMOS+ operations, uses a supervision system called SSC (Communication Supervisory System). This system can monitor, in real time, the network's main parameters, making use of the information supplied by a Supervisory Control and Data Acquisition (SCADA) [24], formed by a Control Center (placed on the main CSE building), and Remote Terminal Units (RTUs) installed into different stations. The use of a SCADA system is due to the management limitations of network communication equipment [25]. Fault identification involves testing the hypothetical faulty components. Repair is achieved by taking intelligent corrective actions.

The SSC allows the operator to acquire information, alarms or digital and analogical parameters of measure, registered on each RTU. Starting from the supplied information, the operator is able to 


\section{Intelligent Integrated Management for Telecommunication Networks}

Antonio Martín, Carlos León, Félix Biscarri

undertake actions through the SSC in order to solve the failures that could appear or to send a technician to repair the stations equipment [26], Figure 7.

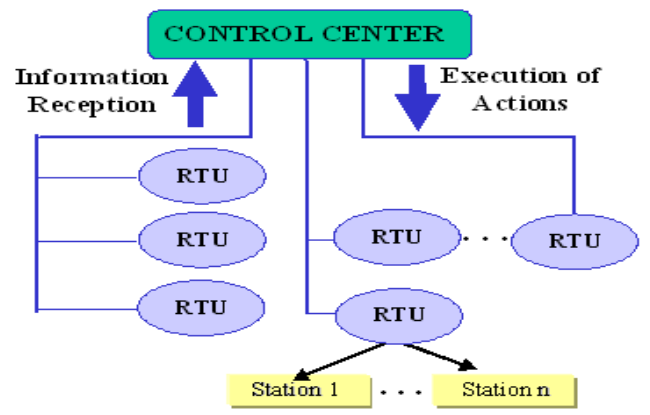

Figure 7. NOMOS+ System Features

The management system in normal operation generates different notifications and alarms. An alarm is an event generated asynchronously whenever the value of some quality indicator crosses a predefined threshold (either positively or negatively). Those alarms are caused when an incident occurs. These events are accompanied by parameters that show different aspects of the events.

\begin{tabular}{|c|}
\hline (31/01 1100.200 stat1 7_TX_C2 stat2 Alarm) \\
\hline (31/01 1103.106 stat1 7_TX_C2 stat2 Alarm_Disappears) \\
\hline (31/01 1122.168 stat1 CTR190/7_RX stat2 Alarm) \\
\hline (31/01 1134.169 Mux3 EXT_FONIA MAD Alarm_Disappears) \\
\hline (31/01 1134.122 stat4 CCA34C_C1C2 stat3 LOCAL_CHANEL_2) \\
\hline (01/02 1034.135 Transc_1 SPU1_BER_1 BER Alarm) \\
\hline (01/02 1034.146 Transc_1 SPU1_BER_1 BER Alarm_Disappears) \\
\hline
\end{tabular}

Each alarm contains information about circumstances that caused the incident [27]. The working memory is where all knowledge is contained each item of knowledge is called a Fact. In a previous relation, taking as an example the third fact, the following information is obtained:

- Date alarm: 31/01.

- Time: 1122.168.

- Alarm kind: CTR190/7_RX. (This case means "Reception error")

- Implied equipments:

- Origin: stat1, "station1".

- Destiny: stat2, "station2".

These events or notifications used, they are previously defined using the corresponding notification template and are including in the same class of managed objects in which the expert rule acts.

When a connection error occurs the device returns the following error messages.

F1 (31/01 1100.200 stat4 7_TX_C2 stat2 ALARM)

F2 (31/01 1103.168 stat4 7_TX_C2 stat2 ALARM)

These alarms indicate problems that require corrective actions. The management system analyzes and checks the rules that match these conditions. If the antecedent of some rule is satisfied, this rule is ready to fire and is placed in system the agenda. When a rule is ready to fire it means that since the antecedent is satisfied, the consequent can be executed. The executed management expert rule in this case is transmissionError. The results generated by the management system are show following: 
FIRE 1: transmissionError f-2

Severity 4

Diagnostic: It damages in the modulate transmission between station 4 and station2.

Recommendation "Revision transceiver

1 rules fired.

Run time is 0.074 seconds, 27.0270 Rules/Sec.

\section{Example of a Management Expert Rule}

Next paragraph shows a complete example of expert rules integration in the GDMO+ proposed standard. It defines a class of managed object named radioTrasceiverCTR190 corresponding to a real device in the network of the power utility.

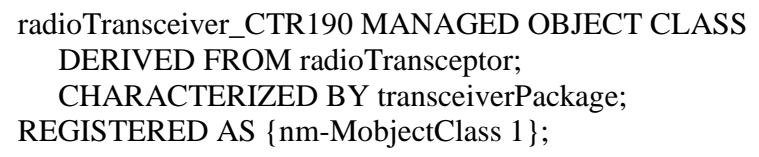

This is a device both sends and receives radio signals. Their primary purpose is to broadcast the signal. The transmitter and the receiver share common circuitry into a single housing like transponders, transverters, and repeaters. These units typically offer the convenient of multiple functions like establishing radio channel, control signals, monitoring station, monitored alarm condition, control logic to activate operations in response to commands received over said communications network, etc.

The class radioTransceiver includes the compulsory transceiverPackage which contains all the specifications corresponding to the device. We can indicate are the three expert rules that have been associated with the defined class by means of the RULES clause.

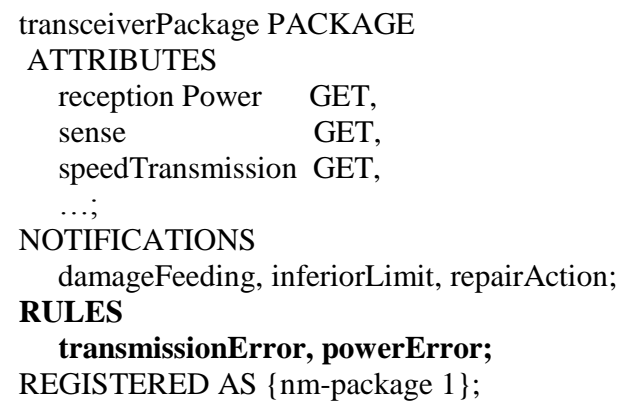

Quite two typical examples of expert rules used in our GDMO Specification are: transmissionError and powerError. These rules are defined by using the RULE template. The expert rules are used within Nomos+ to capture and detect anomalies or defects of operations produced in the transceiver device and suggest the necessary measures for solving the problem.

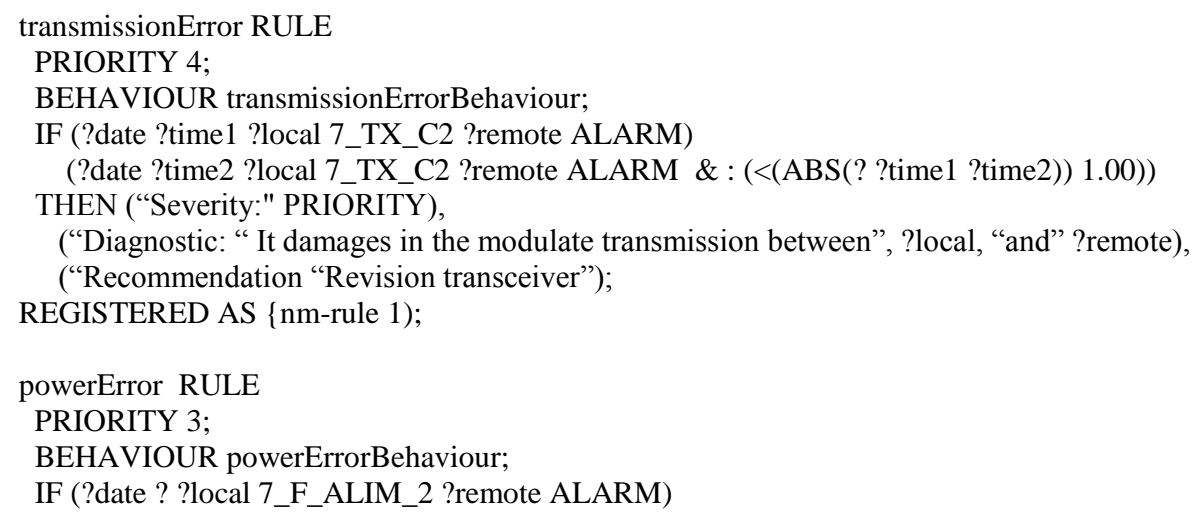


(NOT (?date ? ?local CCA?34_AIS_DE_BB ?remote ALARM))

THEN ("Severity:" PRIORITY),

("Diagnostic: It damages in the electric feeding of the station" ?local),

("Recommendation: To revise the electric connection", ?local);

REGISTERED AS \{nm-rule 2\};

The first rule transmissionError is devoted to the detection of errors in the data transmission module of the transceiver CTR190. The second powerError, is in charge of detecting failures in the power supply of the transceiver CTR190. Both rules give recommendations on how to solve the failures.

\section{Final Prototype Verification}

The purpose is to achieve a functionally correct prototype. Validation constitutes an inherent part of the knowledge based expert system development for NOMOS+ and is intrinsically linked to the development cycle. Validation is essential to the decision-making success of NOMOS+ and to its continued use. An expert system not validated sufficiently may make poor decisions [28]. Validation certainly gives confidence in the system which affects the value of the NOMOS+.

Validation concerns have the following objectives:

- to ascertain what NOMOS+ knows, does not know, or knows incorrectly.

- to ascertain the level of decision expertise of the NOMOS+.

- to determine whether the NOMOS+ is adequately theory based.

- to analyze the reliability of NOMOS+.

To verify the system we feed it with an alarms arbitrary amount. As described, NOMOS+ has been validated with respect to the following aspects: system validation using test cases, validation by case studies, validation against human experts, validation against tough case and validation on site, etc. The result of this proof are including in Table 1.

Table 1. Prototype Testing Results

\begin{tabular}{|c|c|c|c|c|c|c|}
\hline 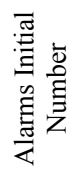 & 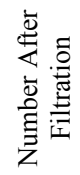 & 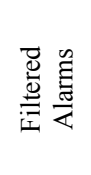 & 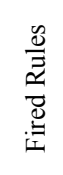 & 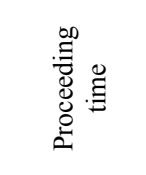 & 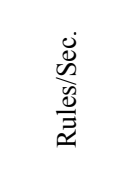 & 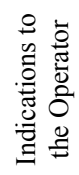 \\
\hline 100 & 1 & 99 & 51 & $0,118 \mathrm{Sec}$. & 432,2034 & 1 \\
\hline 200 & 10 & 95 & 102 & $0,412 \mathrm{Sec}$. & 247,5728 & 6 \\
\hline 300 & 31 & 89,6 & 155 & $1,250 \mathrm{Sec}$. & 124,0000 & 20 \\
\hline 400 & 31 & 92,25 & 201 & 1,438 Sec. & 139,7775 & 16 \\
\hline 500 & 32 & 93,6 & 254 & 2,975 Sec. & 85,3782 & 19 \\
\hline 600 & 38 & 93,66 & 293 & 5,249 Sec. & 55,8202 & 16 \\
\hline 700 & 44 & 93,71 & 346 & $17,982 \mathrm{Sec}$. & 19,2415 & 18 \\
\hline 800 & 55 & 93,125 & 394 & $26,938 \mathrm{Sec}$. & 14,6262 & 23 \\
\hline
\end{tabular}

From these result we can establish the fallowing conclusions:

- Filtration process effectiveness is very high: almost $90 \%$ of the whole. This has the advantage of a decreasing percentage in the amount of indications presented to the operator.

- The speed of the system improves diminishing the number of alarms on which the rest of rules act.

The expert system, with over 200 operation rules, has produced excellent results which, after extensive field-testing, proved to be capable of filtering $90 \%$ of produced alarms with a precision of $95 \%$ in locating them, Figure 8. 


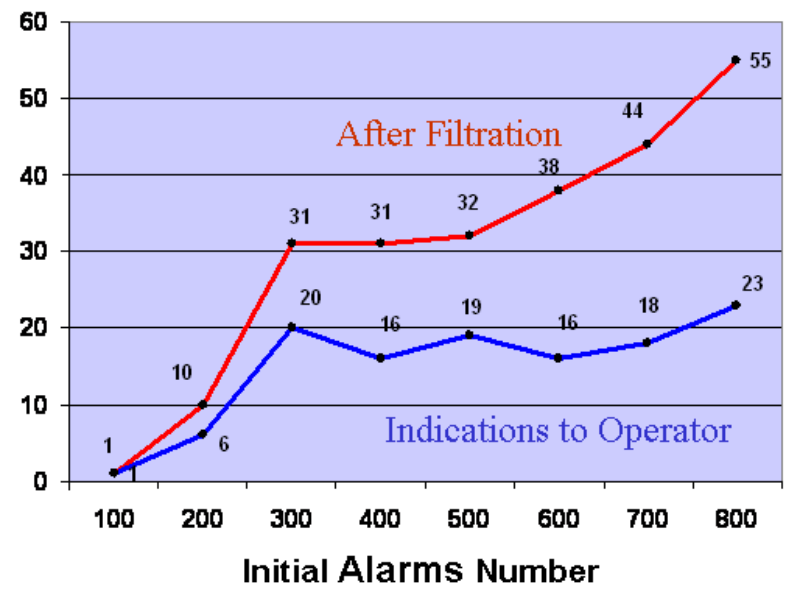

Figure 8. Filtration Process Effectiveness

As noted above, the NOMOS+ performs satisfactorily with about a 95\% rate of success in real cases. The confidence values provided were also found to be in reasonable relative order. It is also noted that the performance of NOMOS+ depends considerably in the facts happened. The more information is input, the better the chance of diagnosing the likely causes of the problems in the network.

\section{Conclusions}

Current networks are very complex and demand ever-increasing levels of quality, making their management a very important aspect to take into account. The traditional model of network administration has certain deficiencies that we have tried to overcome by using a model of intelligent integrated management. To improve the techniques of expert management in a communications network, we propose the possibility of integrating and normalising the expert rules of management within the actual definition of the managed objects. The use of expert agents in network supervision can help the administrator of them in using the maximum capabilities of the network management platform. Intelligent managed objects characteristics are autonomy, reactivity, pro-activeness, mobility and learning.

In this paper we showed possibilities to apply and integrated the artificial intelligence techniques in network management and supervision, using OSI. In fact we believe that these kinds of applications underline the power of GDMO and CMIS as both simple and powerful knowledge modelling and querying languages, offering possibilities that simpler protocols such as SNMP do not offer. We have supplied an original contribution to include expert rules in the specifications of the network elements; for this purpose we have proposed a new standard called Extension of GDMO standard or simply GDMO+. Through the integration of the knowledge within the new extension of the GDMO standard, we can simultaneously define the management information and knowledge. Thus, the management platform is more easily integrated and allows a better adaptation for the network management. Moreover we have built a prototype named NOMOS+ and experiments have been carried out in order to test the efficiency of our proposal.

We conclude pointing out an important aspect of the obtained integration: by using only and exclusively the extended GDMO specification, the administration platform will be able to obtain the management necessary information with respect to the managed objects as well as the expert rules of management that make up the knowledge base of the expert system. This research has demonstrated an useful and interesting modular approach in the development of a knowledge based integrated expert system which can be quite powerful in tackling the huge and enormously wide subject on diagnosis of common problems in management network.

It is suggested that future work should aim to further development of this prototype system by adding more modules based on the framework provided by NOMOS+ so that more in-depth knowledge and specialized subjects may be captured; in particular the following are of great interest: 
Intelligent Integrated Management for Telecommunication Networks Antonio Martín, Carlos León, Félix Biscarri

Development of a design module, possibly a large system, for identifying specific areas: accounting management, configuration management, performance management and security management. Moreover use of external programs and graphics interface to enhance the functions of the system will be desirable. Finally study the possibility of using another method of knowledge representation and reasoning different to the rules: Semantic nets, neuronal nets, frameworks, etc.

\section{References}

[1] M. Douglas and S. Kevin, Schmidt, Essential SNMP, 2nd Edition. O'Reilly, 2005.

[2] ITU-T, Recommendation M.3400, TMN Management Functions. Study Group IV, 1996.

[3] ITU-T Rec. M.3010, Principles for a Telecommunications Management Network (TMN). Study Group IV, 1996.

[4] A. Clemm, Network Management Fundamentals. Cisco Press, 2006.

[5] ITU-T Rec. X.711 Common Management Information Protocol (CMIP) Specification, 1997.

[6] ITU-T Recommendation X.700, Management Framework for Open Systems Interconnection (OSI). CCITT Applications, 1992.

[7] ISO/IEC DIS 10165-4 / ITU-T Recommendation X.722, Information Technology - Open Systems Interconnection - Structure of Management Information - Part 4: Guidelines for the Definition of Managed Objects (GDMO), International Organization for Standardization and International Electrotechnical Committee, 1993.

[8] ITU-T Recommendation X.680, Information technology - Abstract Syntax Notation One (ASN.1): Specification of basic notation, 2002.

[9] Black, U.D., Network Management Standards. McGraw Hill, 1995.

[10] S. Liao, Expert system methodologies and applications - a decade review from 1995 to 2004 , Expert Systems with Applications, 2005.

[11] J. Zuidweg, Next generation intelligent networks. Boston: Artech House, 2002.

[12] R.C. Garcia and J. Cannady, Boundary expansion of expert systems: incorporating evolutionary computation with intrusion detection solutions. SoutheastCon 2001. Proceedings. IEEE, 2001, pp.96-99.

[13] N. Hebrawi, GDMO, Object modelling and definition for network management. Technology appraisals, 1995.

[14] S. B. Morris, Network Management, MIBs and MPLS: Principles, Design and Implementation by Publisher: Addison Wesley, 2003.

[15] ISO/IEC and ITU-T, Information Processing Systems - Open Systems Interconnection - Systems Management Overview. Standard 10040-2, Recommendation X.701, 1998.

[16] D. Udupa, DIVAKARA K. TMN: Telecommunications Management Network. McGraw-Hill, 1999.

[17] C. Baral, Knowledge representation, reasoning, and declarative problem solving. New York: Cambridge University Press, 2003.

[18] R.J. Brachman and H. J. Levesque, representation and reasoning. San Francisco, CA: Elsevier/Morgan Kaufmann, 2004.

[19] J. Luque and F. Gonzalo, Integrating power utility telecommunication networks. Computer Applications in Power, IEEE, 9 (2), 1996, pp. 27-30.

[20] Y. Pencole and M.O. Cordier, A formal framework for the decentralized diagnosis of large scale discrete event systems and its application to telecommunication networks, Artificial Intelligence, 164, 2005, pp. 121-170.

[21] J. Giarratano and G.D. Riley, Expert Systems: Principles and Programming. Book, Brooks/Cole Publishing Co., 2005.

[22] C.H. Wang, S.S. Tseng and T.P. Hong, Integrating membership functions and fuzzy rule sets from multiple knowledge sources, Fuzzy Sets and Systems, 112 (1), 200, pp. 141-154.

[23] M. Burgess, Analytical network and system administration. Managing human-computer networks. Chichester, West Sussex, England ; Hoboken, NJ : John Wiley \& Sons, 2004.

[24] D. Bailey, David, Practical SCADA for industry / David Bailey, Edwin Wright, Newnes, 2006 
[25] R.H. McClanahan, The Benefits of Networked SCADA Systems Utilizing IPEnabled Networks, Rural Electric Power Conference, IEEE, 2002

[26] L. Goleniewski and K.W. Jarrett, Telecommunications Essentials, Second Edition: The Complete Global Source. Addison Wesley Professional, 2006.

[27] W. Stallings, SNMP, SNMPv2, and CMIP: the practical guide to network. Publication Reading, Mass. Addison-Wesley, 2000.

[28] M. Negnevitsky, Artificial intelligence: a guide to intelligent systems. New York: Addison Wesley, 2002. 